\title{
Study of the dose-volume parameters variation in tumor target volumes and organs at risk during nasopharyngeal carcinoma radiotherapy applying deformation registration
}

\author{
Xinsen Yao ${ }^{1,2}$, Guanzhong Gong', Guoping Zuo ${ }^{2}$, Jianxin Ren ${ }^{1}$, Ming Su ${ }^{1,2}$, Yong Yin ${ }^{1}$ \\ ${ }^{1}$ Shandong Cancer Hospital and Institute, Shandong First Medical University and Shandong Academy of Medical Sciences, Ji'nan 250117, China; \\ ${ }^{2}$ School of Nuclear Science and Technology, University of South China, Hengyang 421001, China \\ Contributions: (I) Conception and design: G Gong, Y Yin; (II) Administrative support: Y Yin; (III) Provision of study materials or patients: X Yao; (IV) \\ Collection and assembly of data: X Yao, M Su, J Ren; (V) Data analysis and interpretation: X Yao, G Gong; (VI) Manuscript writing: All authors; (VII) \\ Final approval of manuscript: All authors. \\ Correspondence to: Yong Yin. Shandong Cancer Hospital and Institute, Shandong First Medical University and Shandong Academy of Medical \\ Sciences, No. 440 Jiyan road, Ji’nan 250117, China. Email: yinyongsd@126.com.
}

Background: During intensity-modulated radiotherapy (IMRT) for nasopharyngeal carcinoma (NPC), the volume of the target volume and the organs at risk (OARs) will change constantly, which may lead to differences between the actual dose received and the initial planned dose. In this study, the cumulative dose of the two plans was obtained by deformable registration. This study provides an approach to evaluate the dose volume of IMRT for the NPC objective.

Methods: From July 2014 to May 2018, eighteen NPC patients who accepted simultaneous integrated boost IMRT were enrolled. All patients underwent simulation CT $\left(\mathrm{CT}_{1}\right)$ and replanning $\mathrm{CT}\left(\mathrm{CT}_{2}\right)$ scans after 20-25 fractions of radiation therapy. The treatment plans were designed on $\mathrm{CT}_{1}$ and $\mathrm{CT}_{2}$ with the name of $\mathrm{Plan}_{1}$ and $\mathrm{Plan}_{2}$, respectively. The $\mathrm{Plan}_{\text {reg }}$ and $\mathrm{Plan}_{\text {def }}$ were obtained after registering from $\mathrm{CT}_{2}$ to $\mathrm{CT}_{1}$ using rigidity and deformation technology by Velocity. Then the dose-volume indices of the tumor target volumes and OARs at $\mathrm{Plan}_{1}, \mathrm{Plan}_{2}, \mathrm{Plan}_{\text {rig }}$ and $\mathrm{Plan}_{\text {def }}$ were compared.

Results: The gross tumor volume (GTV) and the left and right parotid gland volumes decreased by $20.8 \%$ $(\mathrm{P}<0.001), 36.8 \%(\mathrm{P}<0.001)$ and 37.5\% $(\mathrm{P}<0.001)$, respectively, from $\mathrm{CT}_{1}$ to $\mathrm{CT}_{2}$. There was no significant difference in the dose-volume index on the GTV and plan gross tumor volume (PGTV) between Plan $_{1}$ and $\mathrm{Plan}_{2}$. The $\mathrm{V}_{30}$ of the left and right parotid gland and the $\mathrm{D}_{\max }$ of the brainstem, left and right eyeballs, left and right lens, and left and right optic nerves were all lower in $\mathrm{Plan}_{2}$ than in $\mathrm{Plan}_{1}$ (the average decrease was $17.0 \%$ to $60.1 \%$ ). The differences in some dose-volume parameters (including $\mathrm{D}_{\text {mean }}$, $\mathrm{D}_{99}$ of the GTV and PGTV, $D_{\text {mean }}$ of the parotid glands, $D_{\text {max }}$ of the lens and optic nerves) between Plan $_{\text {def }}$ and $\operatorname{Plan}_{1}$ were less than $5 \%$. The differences in some dose-volume parameters (including $\mathrm{D}_{\text {mean }}, \mathrm{D}_{95}$ of the GTV and PGTV, $\mathrm{D}_{\text {mean }}, \mathrm{D}_{50}$ and $\mathrm{V}_{30}$ of the parotid glands, $\mathrm{D}_{\max }$ of lens and optic nerves) between Plan $\mathrm{r}_{\mathrm{rig}}$ and $\mathrm{Plan}_{1}$ were less than $10 \%$. The Dyce Similarity Coefficient of the target volume and OARs after deformation registration were higher than that after rigid registration.

Conclusions: The volume of the GTV and parotid glands were decreased during the IMRT for NPC. The dose-volume indices of the GTV and the OARs in Plan $_{\text {def }}$ were similar to those in Plan ${ }_{1}$. Therefore, the dose-volume indices of $\mathrm{Plan}_{1}$ can be used to evaluate the efficacy of radiotherapy and to predict radioactive damage.

Keywords: Nasopharyngeal carcinoma (NPC); image registration technique; dosimetry; radioactive damage

Submitted Jun 24, 2019. Accepted for publication Oct 10, 2019.

doi: $10.21037 /$ tcr.2019.11.06

View this article at: http://dx.doi.org/10.21037/tcr.2019.11.06 


\section{Introduction}

Nasopharyngeal carcinoma (NPC) occurs mostly in Southeast Asia and southern China $(1,2)$. Due to the particularity of its anatomical location, NPC is surrounded by many important tissues and organs. The vast majority of NPC are more sensitive to radioactivity, and consequently radiotherapy or radiotherapy-based comprehensive treatment has now become the main method for radical treatment of patients with NPC (3). For patients with early NPC after radical radiotherapy, the 5-year survival rate can reach $80 \%$.

There are many important organs at risk (OARs) around the target volume of NPC, such as the parotid glands, brain stem, spinal cord, eyeballs, optic nerves and lens. The exposure doses by these OARs were closely related to the quality of life of patients after treatment. The key to improving the effect of radiotherapy is to ensure the dose of the target volume and to minimize the dose of normal tissues and OARs. Therefore, it is of great significance to accurately predict the dose of the target volume and OARs during the radiotherapy process for patients with NPC.

Intensity-modulated radiotherapy (IMRT) is a routine clinical radiotherapy technique for NPC. It has a steep dose gradient distribution, which can reduce the exposure dose of OARs around the tumor while ensuring that the tumor target volume can be irradiated with a sufficient dose (4). However, some studies have shown that the volume and location of tumor target volume and OARs are different during the IMRT for NPC (5-7). In particular, the volume of the parotid glands was significantly reduced during IMRT for NPC (8). Differences in the volume of the parotid glands may lead to inadequate coverage of a radiation dose over the tumor target volume or excessive dosage of OARs, which deviates from the original plan. Deformed registration provides a feasible method for accurately accumulating radiation doses to target volume and OARs in NPC patients during radiotherapy. The purpose of deformed registration technology is to accumulate the dose through point-to-point registration, and then track the dose of the target volume and OARs during IMRT for NPC.

In the course of radiotherapy for NPC, the volume reduction in tumor target and OARs may not be synchronized with the changes in their dose volume indices. This study used registration technology to accumulate the dose of two plans of IMRT for NPC by rigid and deformed methods, and to thereby analyze the difference in the dosevolume index of the tumor target volume and OARs. This study provided an approach to evaluate the dose volume of IMRT for the NPC objective.

\section{Methods}

\section{Patient characteristics}

Eighteen NPC patients (13 males and 5 females) who received simultaneous integrated boost intensity-modulated radiotherapy (SIB-IMRT) from July 2010 to May 2018 were selected. The clinical stage was divided into 10 cases at the $T_{2}$ stage and 8 cases at the $T_{3}$ stage according to the AJCC 2010 staging standard T stage.

\section{CT scanning and planning design}

All patients underwent scanning and simulation on a Philips large-aperture CT, taking the supine position; fixing the head, neck, and shoulder with a thermoplastic mask; and scanning from the top of the head to the lower $3 \mathrm{~cm}$ of the clavicle with a layer thickness of $3 \mathrm{~mm}$. The scanned CT image was transmitted to the Eclipse 13.5 planning system and the SIB-IMRT technique was used. The radiologist outlined the target volume, as well as OARs such as the parotid gland, brain stem, spinal cord, eyeballs, optic nerves, and lens. The dosage limitation conditions for OARs are a $50 \%$ parotid volume dose less than $30 \mathrm{~Gy}$, brainstem dose less than 54 Gy, spinal cord dose less than 45 Gy, optic nerve dose less than $54 \mathrm{~Gy}$, eyeball dose less than $50 \mathrm{~Gy}$, lens dose less than $8 \mathrm{~Gy}$, etc. Plan ${ }_{1}$ was the IMRT plan on simulation CT before radiotherapy and $\mathrm{Plan}_{2}$ was the IMRT plan on repeated simulation $\mathrm{CT}$ at the middle and late stages of radiotherapy. The prescribed dose was 66-70 Gy.

\section{Image registration}

The patient's two radiotherapy plans were imported into the velocity image registration software based on B-spline algorithm, and the repeated simulated $\mathrm{CT}_{2}$ was registered into the first simulated $\mathrm{CT}_{1}$ to obtain the transformed registration image $\mathrm{CT}_{3}$. The dose distribution of $\mathrm{Plan}_{2}$ and the dose distribution of $\mathrm{Plan}_{1}$ were separately subjected to rigidity and deformation registration to obtain $\mathrm{Plan}_{\text {rig }}$ and Plan $_{\text {def }}$.

\section{Statistical indicators}

The volume of the gross tumor volume (GTV) and bilateral 
parotid glands; average dose $\left(\mathrm{D}_{\text {mean }}\right), 95 \%$ volume dose $\left(\mathrm{D}_{95}\right)$ and $99 \%$ volume dose $\left(\mathrm{D}_{99}\right)$ of GTV; and plan gross tumor volume (PGTV) were measured and recorded. Indicators of various OARs include the bilateral parotid $\mathrm{D}_{\text {mean }}, 50 \%$ volume dose $\left(\mathrm{D}_{50}\right)$ and 30 Gy surrounded volume $\left(\mathrm{V}_{30}\right)$; maximum point dose $\left(\mathrm{D}_{\max }\right)$ and $5 \%$ volume dose $\left(\mathrm{D}_{5}\right)$ of the brain stem; $D_{\max }$ of the bilateral eyeballs, optic nerves and lens; and Dice Similarity Coefficient (DSC) of the GTV, PGTV, parotid gland, brainstem, eyeballs, optic nerves and lens after deformation registration and rigid registration. (DSC $=2|A \cap B| /(|A|+|B|)$. The volume of the target volume and OARs in $\mathrm{CT}_{1}$ and $\mathrm{CT}_{2}$ were $\mathrm{A}$ and $\mathrm{B}$ respectively.)

\section{Statistical methods}

The data are expressed in the form $(\bar{x} \pm \mathrm{s})$. A paired $t$-test was performed using SPSS 19.0 statistical software. $\mathrm{P}<0.05$ indicates that the difference is statistically significant.

\section{Results}

\section{Change in the volume of the GTV and parotid gland}

Compared with the initial simulated $\mathrm{CT}_{1}$, the volumes of the GTV, left parotid gland and right parotid gland in the repeated simulated $\mathrm{CT}_{2}$ decreased by $20.83 \%(\mathrm{P}<0.001)$, $36.83 \%(\mathrm{P}<0.001)$ and $37.47 \%(\mathrm{P}<0.001)$, respectively. After deformation registration, the volume of the GTV decreased by $3.15 \%(\mathrm{P}<0.001)$ compared with the initial positioning of the GTV, the left parotid volume decreased by $6.56 \%$ $(\mathrm{P}<0.001)$, and the right parotid volume decreased by $5.89 \%$ $(\mathrm{P}<0.001)$ (Table 1).

\section{Dosimetric comparison in the target volume}

There was no significant change in the dose indices of the GTV and PGTV between $\operatorname{Plan}_{2}$ and $\operatorname{Plan}_{1}(P>0.05)$. Compared with $\mathrm{Plan}_{1}$, the $\mathrm{D}_{\text {mean }}$ of the GTV in $\mathrm{Plan}_{\text {def }}$ and $\mathrm{Plan}_{\text {rig }}$ decreased by $0.42 \%$ and $0.62 \%$, respectively (Table 2), and the difference was statistically significant $(\mathrm{P}<0.05)$. The $\mathrm{D}_{\text {mean }}$ of the PGTV in $\mathrm{Plan}_{\text {def }}$ and Plan $\mathrm{Pl}_{\text {rig }}$ decreased by $1.08 \%$ and $1.49 \%$, respectively; the $\mathrm{D}_{95}$ of the PGTV in $\mathrm{Plan}_{\text {def }}$ and $\operatorname{Plan}_{\text {rig }}$ decreased by $2.77 \%$ and $5.49 \%$, respectively; the $\mathrm{D}_{99}$ of the PGTV in $\mathrm{Plan}_{\text {def }}$ and $\mathrm{Plan}_{\text {rig }}$ decreased by $4.29 \%$ and $7.39 \%$ (Table 2); and these differences were statistically significant $(\mathrm{P}<0.05)$.

\section{DSC comparison between rigid registration and deformation registration}

The DSC of the target volume and the OARs after rigid registration and deformation registration, respectively, is shown in Table 3. The DSC of the target volume and the OARs after deformation registration was greater than the DSC of the rigid registration target volume and the OARs. The DSC difference between the left and right parotid deformation registration and rigid registration was significant $(\mathrm{P}<0.05)$.

\section{Dosimetric comparison in the OARs}

The dose distribution of each OAR in $\mathrm{Plan}_{1}, \mathrm{Plan}_{2}, \mathrm{Plan}_{\text {def }}$ and $\mathrm{Plan}_{\text {rig }}$ is shown in Table 4. Compared with $\operatorname{Plan}_{1}$, the $\mathrm{V}_{30}$ of the left parotid gland in $\mathrm{Plan}_{2}$ decreased by $33.5 \%$, the $\mathrm{D}_{\text {mean }}$ of the right parotid gland decreased by $31.45 \%$, the $\mathrm{V}_{30}$ decreased by $60.13 \%$, and the $\mathrm{D}_{50}$ decreased by $28.35 \%$. The difference was statistically significant $(\mathrm{P}<0.05)$. The $\mathrm{D}_{\text {mean }}$ of the left and right parotid glands in Plan $_{\text {def }}$ decreased by $1.98 \%$ and $2.3 \%$, respectively, compared with $\mathrm{Plan}_{1}$, and the difference was statistically significant $(\mathrm{P}<0.05)$. The changes in $V_{30}$ and $D_{50}$ of the left and right parotid glands between $\mathrm{Plan}_{\text {def }}$ and $\mathrm{Plan}_{1}$ were not significant $(\mathrm{P}>0.05)$. The $\mathrm{D}_{\text {mean }}$ of the left and right parotid in Plan $_{\text {rig }}$ decreased by $4.21 \%$ and $4.93 \%$ compared with $\operatorname{Plan}_{1}, \mathrm{~V}_{30}$ decreased by $7.68 \%$ and $10.35 \%$, and $\mathrm{D}_{50}$ decreased by $3.37 \%$ and $3.46 \%$, and this difference was statistically significant $(\mathrm{P}<0.05)$.

Compared with $\mathrm{Plan}_{1}, \mathrm{D}_{\max }$ and $\mathrm{D}_{5}$ of the brainstem in $\mathrm{Plan}_{2}$ were reduced by $17.04 \%$ and $27.3 \%$, respectively. The $\mathrm{D}_{\max }$ of the left eyeball, right eyeball, left lens, right lens, left optic nerve and right optic nerve were decreased by $36.43 \%, 28.48 \%, 24.95 \%, 24.29 \%, 44.69 \%$ and $41.1 \%$, respectively. The difference was statistically significant $(\mathrm{P}<0.05)$. Compared with $\mathrm{Plan}_{1}, \mathrm{D}_{5}$ of the brainstem in Plan $_{\text {def }}$ was reduced by $3.3 \%$. The $\mathrm{D}_{\text {max }}$ of the left eyeball, right eyeball, left optic nerve and right optic nerve were decreased by $6.13 \%, 3.44 \%, 5.67 \%$ and $5.19 \%$, respectively. The difference was statistically significant $(\mathrm{P}<0.05)$.

Compared with $\mathrm{Plan}_{\text {rig }}$, the $\mathrm{D}_{\text {mean }}, \mathrm{V}_{30}$ and $\mathrm{D}_{50}$ of the left parotid gland in $\mathrm{Plan}_{\text {def }}$ increased by $2.26 \%, 4.36 \%$ and $2.1 \%$, respectively, and the difference was statistically significant $(\mathrm{P}<0.05)$. The difference in $\mathrm{D}_{\text {mean }}, \mathrm{V}_{30}$ and $\mathrm{D}_{50}$ in the right parotid gland was not significant. The $\mathrm{D}_{\max }$ of the OARs, such as the brainstem, eyeballs, lens and optic nerves, were all decreased. 
Table 1 Volume of the tumor target volume and parotid gland in positioning image $\mathrm{CT}_{1}$, reset image $\mathrm{CT}_{2}$ and registration image $\mathrm{CT}_{3}\left(\mathrm{~cm}^{3}, \bar{x}_{ \pm}\right)$

\begin{tabular}{|c|c|c|c|c|c|c|c|}
\hline Parts & $\mathrm{CT}_{1}$ & $\mathrm{CT}_{2}$ & $\mathrm{CT}_{3}$ & \multicolumn{2}{|c|}{$\mathrm{CT}_{1} \& \mathrm{CT}_{2}$} & \multicolumn{2}{|c|}{$\mathrm{CT}_{1} \& \mathrm{CT}_{3}$} \\
\hline GTV & $79.28 \pm 49.66$ & $64.47 \pm 42.39$ & $76.79 \pm 48.21$ & 5.477 & 0.000 & 4.292 & 0.000 \\
\hline Parotid-L & $16.76 \pm 4.74$ & $10.77 \pm 4.07$ & $15.61 \pm 4.43$ & 9.972 & 0.000 & 5.735 & 0.000 \\
\hline Parotid-R & $16.63 \pm 4.40$ & $10.46 \pm 3.99$ & $15.58 \pm 4.03$ & 9.303 & 0.000 & 6.116 & 0.000 \\
\hline
\end{tabular}

Table 2 Target volume the dose distribution (Gy, $\bar{x} \pm \mathrm{s})$

\begin{tabular}{llccccccccccccc}
\hline Target & Parameter & Plan $_{1}$ & Plan $_{2}$ & Plan $_{\text {def }}$ & Plan $_{\text {rig }}$ & $\mathrm{t}_{1}$ & $\mathrm{P}_{1}$ & $\mathrm{t}_{2}$ & $\mathrm{P}_{2}$ & $\mathrm{t}_{3}$ & $\mathrm{P}_{3}$ & $\mathrm{t}_{4}$ & $\mathrm{P}_{4}$ \\
\hline GTV & $\mathrm{D}_{\text {mean }}$ & $73.91 \pm 3.59$ & $73.85 \pm 3.55$ & $73.60 \pm 3.56$ & $73.45 \pm 3.54$ & 0.533 & 0.601 & 2.714 & 0.015 & 3.308 & 0.004 & 1.553 & 0.139 \\
& $\mathrm{D}_{95}$ & $71.93 \pm 3.49$ & $71.79 \pm 3.91$ & $71.35 \pm 3.78$ & $70.51 \pm 4.13$ & 0.853 & 0.405 & 1.792 & 0.091 & 2.361 & 0.030 & 1.432 & 0.170 \\
& $\mathrm{D}_{99}$ & $71.21 \pm 3.50$ & $70.29 \pm 4.96$ & $70.21 \pm 3.87$ & $67.60 \pm 8.36$ & 1.398 & 0.180 & 2.516 & 0.022 & 1.948 & 0.068 & 1.470 & 0.160 \\
\multirow{2}{*}{ PGTV } & $\mathrm{D}_{\text {mean }}$ & $73.56 \pm 3.42$ & $72.10 \pm 6.48$ & $72.78 \pm 3.70$ & $72.48 \pm 3.71$ & 1.053 & 0.307 & 3.935 & 0.001 & 3.734 & 0.002 & 1.555 & 0.138 \\
& $\mathrm{D}_{95}$ & $70.99 \pm 3.43$ & $69.14 \pm 6.46$ & $69.09 \pm 5.12$ & $67.12 \pm 7.47$ & 1.313 & 0.207 & 3.319 & 0.004 & 2.553 & 0.021 & 1.441 & 0.168 \\
& $\mathrm{D}_{99}$ & $69.08 \pm 3.60$ & $65.85 \pm 7.75$ & $66.23 \pm 6.29$ & $64.03 \pm 8.63$ & 1.863 & 0.080 & 3.195 & 0.005 & 2.819 & 0.012 & 1.657 & 0.116 \\
\hline
\end{tabular}

$\mathrm{t}_{1}-\mathrm{t}_{4}, \mathrm{P}_{1}-\mathrm{P}_{4}$ represent the values of $\mathrm{Plan}_{1}$ and Plan $_{2}, \mathrm{Plan}_{1}$ and Plan def, Plan $_{1}$ and Plan $_{\text {rig }}$, and Plan $_{\text {def }}$ and Plan rig as paired $t$-tests, respectively.

Table 3 DSC for the deformation registration and rigid registration

\begin{tabular}{lcccc}
\hline Parts & Deformation DSC & Rigid DSC & $\mathrm{t}$ & $\mathrm{P}$ \\
\hline GTV & $0.76 \pm 0.10$ & $0.73 \pm 0.10$ & 1.744 & 0.099 \\
PGTV & $0.77 \pm 0.13$ & $0.75 \pm 0.12$ & 1.550 & 0.139 \\
Parotid-L & $0.70 \pm 0.09$ & $0.56 \pm 0.16$ & 5.158 & 0.000 \\
Parotid-R & $0.71 \pm 0.10$ & $0.58 \pm 0.12$ & 7.808 & 0.000 \\
Brainstem & $0.85 \pm 0.04$ & $0.85 \pm 0.04$ & -0.368 & 0.718 \\
Eye-L & $0.87 \pm 0.05$ & $0.84 \pm 0.07$ & 1.971 & 0.065 \\
Eye-R & $0.87 \pm 0.06$ & $0.82 \pm 0.09$ & 1.985 & 0.064 \\
Lens-L & $0.50 \pm 0.24$ & $0.44 \pm 0.24$ & 1.044 & 0.311 \\
Lens-R & $0.51 \pm 0.25$ & $0.43 \pm 0.26$ & 1.464 & 0.162 \\
Optic-L & $0.61 \pm 0.17$ & $0.49 \pm 0.22$ & 2.664 & 0.016 \\
Optic-R & $0.57 \pm 0.14$ & $0.50 \pm 0.19$ & 2.001 & 0.062 \\
\hline Spinal cord & $0.83 \pm 0.03$ & $0.68 \pm 0.13$ & 4.660 & 0.000
\end{tabular}

\section{Discussion}

In the process of IMRT, although IMRT is more effective than conventional radiotherapy in increasing the target dose, the radiation exposure of normal tissues and OARs is relatively low $(9,10)$. However, for NPC, factors such as changes in the patient weight, volume of tumors and OARs may have a certain impact on dose assessment. Anatomical changes may result in an insufficient target dose and excessive exposure of OARs. The difference between the actual dose received by the patient and the radiotherapy plan designed by simulation CT was $15 \%$ (11). Lu et al. (12) compared the repeated simulated CT with the initial simulated CT in 12 patients with NPC and found that the volume of the PGTV was reduced by an average of $16.4 \% \pm 27.3 \%$. In this study, the tumor target volume of repeated simulated CT was reduced by $20.83 \%$ on average compared with that of the initial simulated CT, and the difference between the Plan deformation, $\mathrm{Plan}_{2}$ and $\mathrm{Plan}_{1}$ dose evaluation showed that the $\mathrm{D}_{\text {mean }}, \mathrm{D}_{95}$ and $\mathrm{D}_{99}$ of the GTV were not significant. Cheng et al. (13) and Wang et al. (14) showed that in the course of radiotherapy for NPC, the volume of the tumor target was smaller than that before radiotherapy, but the target dose index did not decrease. In this study, it was found that the volume of the target gradually decreased during IMRT for NPC, and this conclusion is consistent with most studies (12-14). The dose index of the target volume was basically the same in the four plans. Therefore, the dose index of target volume in the initial plan can accurately evaluate the efficacy of radiotherapy. 
Table 4 Dose distribution of OARs $(x \pm s)$

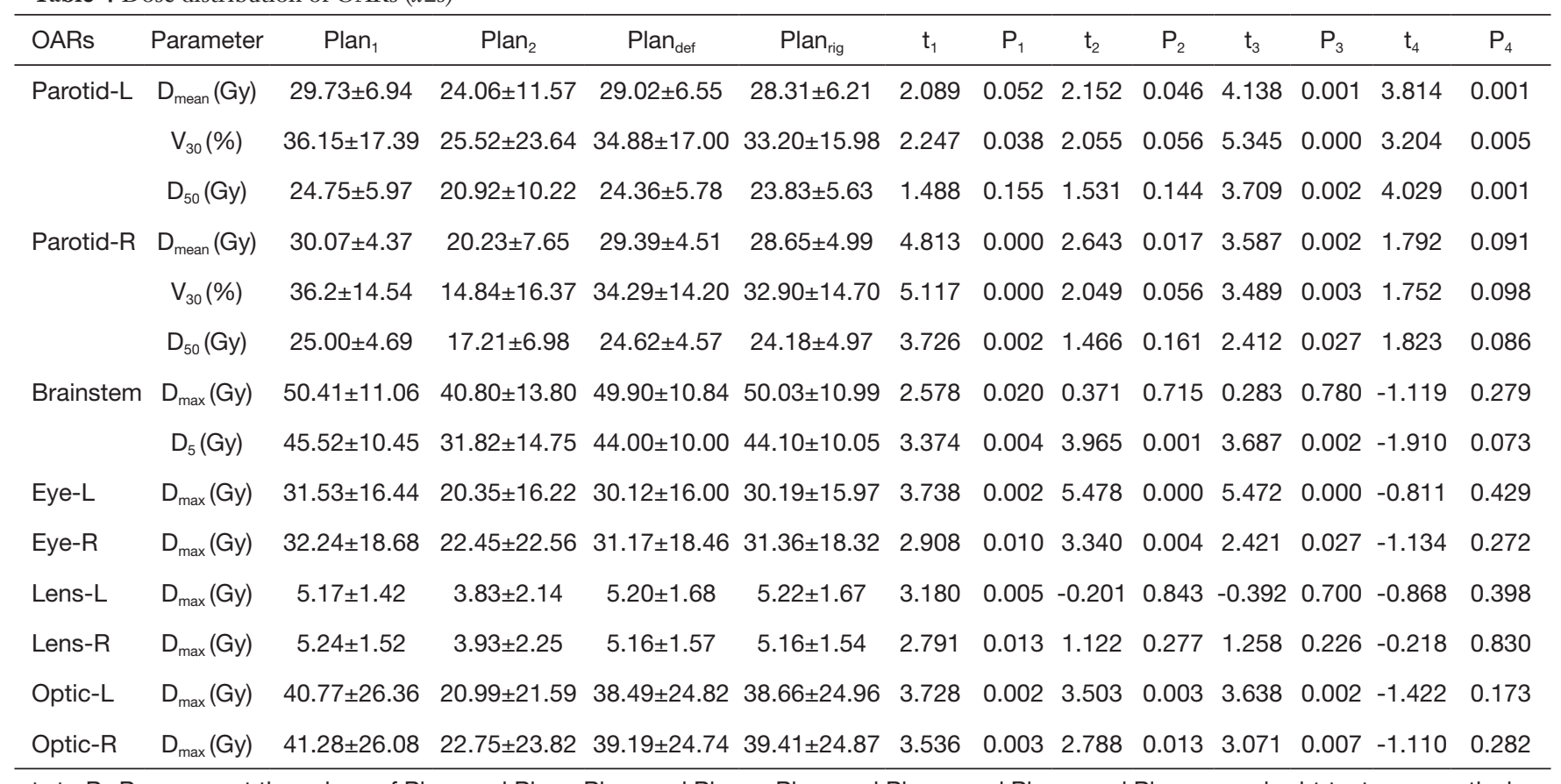

$\mathrm{t}_{1}-\mathrm{t}_{4}, \mathrm{P}_{1}-\mathrm{P}_{4}$ represent the values of $\mathrm{Plan}_{1}$ and $\mathrm{Plan}_{2}, \mathrm{Plan}_{1}$ and Plan def, Plan $_{1}$ and $\mathrm{Plan}_{\text {rig }}$, and Plan $_{\text {def }}$ and Plan rig $_{\text {rig }}$ as paired $t$-tests, respectively.

The parotid gland is sensitive to radiation, and the volume of the parotid gland changes significantly during radiotherapy for NPC. Fung et al. (15) transmitted the original plan dose distribution of NPC radiotherapy to the new CT to form a synthetic plan compared with the original plan dosimetry, the dose of the OARs increased, and the $\mathrm{D}_{\text {mean }}$ of the left and right parotid glands significantly increased. In this study, we found that the volume of the parotid gland changed significantly compared with the initial location CT, and the left and right parotid glands were reduced by $36.83 \%$ and $37.47 \%$, respectively. Compared with $\mathrm{Plan}_{1}$, the $\mathrm{V}_{30}$ of the left parotid gland in Plan $_{2}$ decreased by $36.15 \%$; the $\mathrm{D}_{\text {mean }}, \mathrm{V}_{30}$ and $\mathrm{D}_{50}$ of the right parotid gland decreased by $30.07 \%, 36.2 \%$ and $25 \%$, respectively. The dose-volume evaluation of $\mathrm{Plan}_{\text {def }}$ and $\mathrm{Plan}_{1}$ showed that the $\mathrm{D}_{\text {mean }}$ of the left and right parotid glands decreased by $1.98 \%$ and $2.3 \%$, respectively, and the $V_{30}$ and $D_{50}$ of the left and right parotid glands were not statistically significant. These studies have shown that the parotid gland volume shrinks as the dose is absorbed during radiotherapy, and the proportion of shrinkage is not synchronized with the shrinkage of the tumor target volume. In this study, it was found that the dose-volume index of parotid glands in $\mathrm{Plan}_{1}$ and $\mathrm{Plan}_{2}$ was significantly different. The dose-volume index of $\mathrm{Plan}_{1}$ and $\mathrm{Plan}_{2}$ may not accurately predict the radiation damage of parotid glands during radiotherapy. However, deformation accumulation is an objective method to eliminate the effect of volume shape change, and the difference in the dosevolume index between the $\mathrm{Plan}_{1}$ and $\mathrm{Plan}_{\text {def }}$ were less than $5 \%$. Therefore, it can be considered that the use of the initial planned dose-volume index can accurately predict the radiation damage of the parotid gland during IMRT for NPC.

Compared with the initial simulated CT, repeated simulated CT was deformed to different degrees, and therefore, the dose distribution of the two plans cannot be accumulated directly. Image registration method is needed to deform and accumulate the dose. Janssens et al. (16) used the Demons algorithm based on the image grayscale and the Morphons algorithm based on the edge and line to achieve the deformation registration. In this study, deformation registration and rigid registration based on the $\mathrm{B}$-spline algorithm were adopted. The DSC of the target volume and OARs in the deformation registration were larger than that in the rigid registration, especially with respect to the parotid glands $(\mathrm{P}<0.05)$, which shows that deformation registration was superior to rigid registration.

The volume changes in the brain stem, eyeballs, optic nerves, and lens during IMRT for NPC were very small. 
Compared with $\mathrm{Plan}_{1}, \mathrm{Plan}_{2}$ showed a significant difference $(\mathrm{P}<0.05)$ in the dose index of these OARs, which was attributed to the shrinking of the GTV volume, and the variation in the dose-volume index in $\mathrm{Plan}_{\text {def }}$ compared with $\mathrm{Plan}_{1}$ were very small.

\section{Conclusions}

In summary, during IMRT for NPC, the volume of the tumor target volume and parotid gland were significantly reduced. However, there was no significant difference between the dose-volume index of the tumor target and OARs in the first planning and the dose-volume index accumulated by deformation registration. Therefore, the dose-volume index of the initial plan can be used to evaluate the curative effect of NPC patients in the whole radiotherapy process and to predict the radiation damage.

\section{Acknowledgments}

Funding: This research was supported by the Key Research and Development Program of Guangxi Province (No. $\mathrm{AB} 17195005)$ and the Key Research and Development Program of Shandong Province (No. 2018GSF118006).

\section{Footnote}

Conflicts of Interest: All authors have completed the ICMJE uniform disclosure form (available at http://dx.doi. org/10.21037/tcr.2019.11.06). The authors have no conflicts of interest to declare.

Ethical Statement: The authors are accountable for all aspects of the work in ensuring that questions related to the accuracy or integrity of any part of the work are appropriately investigated and resolved. The study was conducted in accordance with the Declaration of Helsinki (as revised in 2013). This retrospective study was approved by the Institutional Review Board (IRB) and the Ethics Committee of the Shandong Cancer Hospital, and the number/ID of the approval 201806033. Individual informed consent was waived due to the retrospective nature of the study.

Open Access Statement: This is an Open Access article distributed in accordance with the Creative Commons Attribution-NonCommercial-NoDerivs 4.0 International License (CC BY-NC-ND 4.0), which permits the non- commercial replication and distribution of the article with the strict proviso that no changes or edits are made and the original work is properly cited (including links to both the formal publication through the relevant DOI and the license). See: https://creativecommons.org/licenses/by-nc-nd/4.0/.

\section{References}

1. Zeng L, Sun XM, Chen CY, et al. Comparative study on prophylactic irradiation to the whole neck and to the upper neck for patients with neck lymph node negative nasopharyngeal carcinoma. Head Neck 2014;36:687-93.

2. Zhang L, Chen QY, Liu H, et al. Emerging treatment options for nasopharyngeal carcinoma. Drug Des Devel Ther 2013;7:37-52.

3. Wei WI, Sham JST. Nasopharyngeal carcinoma. Lancet 2005;365:2041-54.

4. Lai SZ, Li WF, Chen L, et al. How does intensitymodulated radiotherapy versus conventional twodimensional radiotherapy influence the treatment results in nasopharyngeal carcinoma patients? Int J Radiat Oncol Biol Phys 2011;80:661-8.

5. Zhao L, Wan Q, Zhou Y, et al. The role of replanning in fractionated intensity modulated radiotherapy for nasopharyngeal carcinoma. Radiother Oncol 2011;98:23-7.

6. Wang RH, Zhang SX, Zhou LH, et al. Volume and dosimetric variations during two-phase adaptive intensity-modulated radiotherapy for locally advanced nasopharyngeal carcinoma. Biomed Mater Eng 2014;24:1217-25.

7. Orlandi E, Tomatis S, Potepan P, et al. Critical analysis of locoregional failures following intensity-modulated radiotherapy for nasopharyngeal carcinoma. Future Oncol 2013;9:103-14.

8. Zhou N, Chu C, Dou X, et al. Early evaluation of radiation-induced parotid damage in patients with nasopharyngeal carcinoma by $\mathrm{T} 2$ mapping and mDIXON Quant imaging: initial findings. Radiat Oncol 2018;13:22.

9. Deng S, Liu X, Lu H, et al. Three-Phase Adaptive Radiation Therapy for Patients with Nasopharyngeal Carcinoma Undergoing Intensity-Modulated Radiation Therapy: Dosimetric Analysis. Technol Cancer Res Treat 2017;16:910-6.

10. Zhang $\mathrm{Y}, \mathrm{Lin} \mathrm{C}, \mathrm{Wu} \mathrm{J}$, et al. A longitudinal evaluation of early anatomical changes of parotid gland in intensity modulated radiotherapy of nasopharyngeal carcinoma patients with parapharyngeal space involvement. J Med Radiat Sci 2017;64:188-94. 
11. Lee C, Langen KM, Lu W, et al. Assessment of Parotid Gland Dose Changes During Head and Neck Cancer Radiotherapy Using Daily Megavoltage Computed Tomography and Deformable Image Registration. Int J Radiat Oncol Biol Phys 2008;71:1563-71.

12. Lu J, Ma Y, Chen J, et al. Assessment of anatomical and dosimetric changes by a deformable registration method during the course of intensity-modulated radiotherapy for nasopharyngeal carcinoma. J Radiat Res 2014;5 5:97-104.

13. Cheng HC, Wu VW, Ngan RK, et al. A prospective study on volumetric and dosimetric changes during intensity-

Cite this article as: Yao X, Gong G, Zuo G, Ren J, Su M, Yin Y. Study of the dose-volume parameters variation in tumor target volumes and organs at risk during nasopharyngeal carcinoma radiotherapy applying deformation registration. Transl Cancer Res 2019;8(8):2886-2892. doi: 10.21037/tcr.2019.11.06 modulated radiotherapy for nasopharyngeal carcinoma patients. Radiother Oncol 2012;104:317-23.

14. Fung $W W$, Teo PM, Wu VW. Developing an adaptive radiation therapy strategy for nasopharyngeal carcinoma. J Radiat Res 2014;55:293-304.

15. Fung WW, Wu VW, Teo PM. Dosimetric evaluation of a three-phase adaptive radiotherapy for nasopharyngeal carcinoma using helical tomotherapy. Med Dosim 2012;37:92-7.

16. Janssens G, Xivry JOD, Fekkes S, et al. Evaluation of nonrigid registration models for interfraction dose accumulation in radiotherapy. Med Phys 2009;36:4268-76. 